Research Article

\title{
Metabolomics Approach for Discrimination and Quality Control of Natural and Commercial Fallopia multiflora Products in Vietnam
}

\author{
Nguyen Thi Thoa, ${ }^{1,2}$ Nguyen Hai Dang $\mathbb{D}^{3},{ }^{3}$ Do Hoang Giang $\mathbb{D},{ }^{4}$ Nguyen Thi Thu Minh, ${ }^{4}$ \\ and Nguyen Tien Dat $\mathbb{D}^{1,4}$ \\ ${ }^{1}$ Graduate University of Science and Technology, Vietnam Academy of Science and Technology, 18-Hoang Quoc Viet, Cau Giay, \\ Hanoi, Vietnam \\ ${ }^{2}$ Faculty of Chemical Technology, Hanoi University of Industry, 298-Cau Dien, Bac Tu Liem, Hanoi, Vietnam \\ ${ }^{3}$ University of Science and Technology of Hanoi, Vietnam Academy of Science and Technology, 18-Hoang Quoc Viet, Cau Giay, \\ Hanoi, Vietnam \\ ${ }^{4}$ Center for Research and Technology Transfer, Vietnam Academy of Science and Technology, 18-Hoang Quoc Viet, Cau Giay, \\ Hanoi, Vietnam
}

Correspondence should be addressed to Nguyen Tien Dat; ngtiend@imbc.vast.vn

Received 11 April 2020; Revised 27 August 2020; Accepted 26 October 2020; Published 6 November 2020

Academic Editor: Günther K. Bonn

Copyright (c) 2020 Nguyen Thi Thoa et al. This is an open access article distributed under the Creative Commons Attribution License, which permits unrestricted use, distribution, and reproduction in any medium, provided the original work is properly cited.

\begin{abstract}
A precise HPLC-DAD-based quantification together with the metabolomics statistical method was developed to distinguish and control the quality of Fallopia multiflora, a popular medicinal material in Vietnam. Multivariate statistical methods such as hierarchical clustering analysis and principal component analysis were utilized to compare and discriminate six natural and twelve commercial samples. 2,3,4',5-Tetrahydroxystilbene 2-O- $\beta$-D-glucopyranoside (THSG) (1), emodin (4), and the new compound 6hydroxymusizin 8 -O- $\alpha$-D-apiofuranosyl-(1 $\longrightarrow 6)-\beta$-D-glucopyranoside $(5)$ could be considered as important markers for classification of F. multiflora. Furthermore, seven phenolics were quantified that the variation in the contents of selected metabolites revealed the differences in the quality of natural and commercial samples. Recovery of the compounds from the analytes was more than $98 \%$, while the limits of detection (LOD) and the limits of quantitation (LOQ) ranged from 0.5 to $6.6 \mu \mathrm{g} / \mathrm{ml}$ and 1.5 to $19.8 \mu \mathrm{g} / \mathrm{ml}$, respectively. The linearity, LOD, LOQ, precision, and accuracy satisfied the criteria FDA guidance on bioanalytical methods. Overall, this method is a promising tool for discrimination and quality assurance of $F$. multiflora products.
\end{abstract}

\section{Introduction}

Fallopia multiflora (Thunberg) Haraldson, synonym Polygonum multiflorum Thunb., is a well-known traditional medicine in several South Asian countries. The roots of this plant have been used to promote hair growth and enhance liver and kidney functions [1]. Previous studies reported that the major compounds, i.e., 2,3,4',5-tetrahydroxystilbene 2 $O-\beta$-D-glucopyranoside (THSG) and emodin may exhibit hepatoprotective $[2-4]$, antiaging $[5,6]$, and antioxidant activities [7]. THSG and other stilbenes also showed the antihyperlipidemia effect [8], whereas emodin exhibited significant anticancer, anti-inflammatory, and tonic tension inhibiting effects $[9,10]$. Also, polyphenolics, flavonoids, glycosides, tannins, steroids, and other types of compounds were isolated from F. multiflora which have shown remarkable antioxidant, immunomodulating, and cerebrovascular protective effects [9-14]. Commonly, roots of F. multiflora are processed by steaming with black beans, thus, declining more than half of THSG content [15]. Recent studies showed that the contents of THSG, emodin, emodin8-O-glucoside, and gallic acid could be decreased sharply at high temperature, hence, decreased its antioxidant activity $[16,17]$. The phytochemicals of $F$. multiflora may vary due to 
geographical origins. Several recent studies demonstrated that the contents of THSG, emodin, and physcion might be dramatically different due to the origins of the plants [18-20]. HPLC-fingerprint chromatography and LC-MS based metabolomics approaches are the common methods for geographical and processing discrimination of F. multiflora, which illustrated clearly the markers to distinguish the samples with high accuracy $[21,22]$.

In Vietnam, F. multiflora has been widely used in raw materials or processed products [23]. However, there is no effective way to classify, identify, and control the quality of commercial F. multiflora products in Vietnam. Recently, we reported about the phenolic constituents from F. multiflora roots, including THSG (1), physcionin (2), emodin 8-glucoside (3), and emodin (4), pleuropyrone A (6), torachrysone 8-O- $\beta$-D-glucopyranoside (7), and the naptholic derivative 6-hydroxymusizin $\quad 8-O-\alpha$-D-apiofuranosyl$(1 \longrightarrow 6)-\beta$-D-glucopyranoside (5) (Figure 1) [24]. In the present study, we performed HPLC analyses on the phenolic contents and applied the statistical methods to discriminate the natural roots and commercial medicinal slices of F. multiflora. In addition, the contents of these compounds were quantified and compared among the samples.

\section{Materials and Methods}

2.1. Sample Preparation. Six samples of 3- to 4-year-old natural roots of F. multiflora, coded Nat_1 to Nat_6, were collected in six districts of Ha Giang, a northern mountainous province in Vietnam, in October 2015. These samples were identified by Dr. Nguyen The Cuong, the Institute of Ecology and Biological Resources, Vietnam Academy of Science and Technology. Commercial medicinal slices (CMS) of F. multiflora, which coded CMS_1 to CMS_12 were purchased at oriental medicine stores in Hanoi, Vietnam, in November 2015. After being cut and powdered, $5 \mathrm{~g}$ of each sample was extracted three times in methanol under sonication condition at $30^{\circ} \mathrm{C}$. Then, all of the filtrates were concentrated and made up the volume with methanol to $20 \mathrm{ml}$. The samples could be diluted if necessary. All solutions had been filtered through $0.45 \mu \mathrm{m}$ Agilent Econofilters (Agilent Technologies, USA) before being injected to the HPLC system. Each sample was analyzed in triplicate.

2.2. Prepare Calibration Standards. Seven standard compounds (Figure 1) were isolated from F. multiflora as described in the previous paper [24]. Purities of these compounds were assessed at more than $95 \%$ by HPLC analyses (data not shown). Calibration solutions for each standard compound were prepared at the concentration range of $5-1000 \mu \mathrm{g} / \mathrm{ml}$. Quality control samples for the compounds were prepared at $25 \mu \mathrm{g} / \mathrm{ml}, 125 \mu \mathrm{g} / \mathrm{ml}$, and $500 \mu \mathrm{g} / \mathrm{ml}$.

2.3. Instrumentation. HPLC-grade solvents were obtained from Scharlau (Spain) and Merck Millipore (Darmstadt, Germany). HPLC-DAD data were obtained on an Agilent
Series 1260 (Agilent Technologies, USA) system, consisting of a vacuum degasser, a quaternary mixing pump, an autosampler, a column oven, and a diode-array detector (DAD) using a ZORBAX Eclipse XDB C18 column (150 mm x 4,6 mm, $5 \mu \mathrm{m}$ ) equipped with a ZORBAX Eclipse XDB C18 guard column $(12.5 \mathrm{~mm} \times 4.6 \mathrm{~mm}, 5 \mu \mathrm{m})$ (Agilent Technologies, USA). A mixture of $0.1 \% \mathrm{v} / \mathrm{v}$ of acetic acid in water (mobile phase A) and methanol $(\mathrm{MeOH})$ (mobile phase B) was utilized as the mobile phase.

2.4. Linearity, $L O D$, and $L O Q$. Linearity was evaluated in this concentration range three separate times. The calibration curve obtained had to have a correlation coefficient (R2) at least 0.999 for every analyte. The limit of detection (LOD) was the lowest concentration of the standard compound possibly identified. Meanwhile, the limit of quantitation (LOQ) illustrated the lowest concentration of analyte possibly quantified. LOD and LOQ were obtained using the calibration curve, based on the standard deviation (SD) of the response and the slope of the calibration curve, according to the equations as follows:

$$
\begin{aligned}
& \text { LOD }=3.3 \frac{\sigma}{\mathrm{a}}, \\
& \text { LOQ }=10 \frac{\sigma}{\mathrm{a}},
\end{aligned}
$$

where $\sigma$ is the standard derivation of the data response, and a is the slope of the calibration curve.

2.5. Precision and Accuracy. The intraday of variation was evaluated by analyzing the response of six replicates of the low, medium, and high concentrations of quality control samples at 25,125 , and $500 \mu \mathrm{g} / \mathrm{ml}$, respectively, on the same day, whereas the interday variation was evaluated with those quality control samples injected into the HPLC over three consecutive days. The precision of the measurement was assessed by the coefficient of variation (CV\%), which was calculated by the ratio of standard deviations of replicates to the mean concentrations. The accuracy was calculated from the recovery of the standard spiking into a sample that had known the contents of the compounds. The mixture was extracted and analyzed using the method mentioned above. Six replicates were performed for the test.

2.6. Data Processing and Statistical Analysis. Data acquisition and processing were performed by Agilent ChemStation (Agilent Technologies, USA) and Microsoft Excel 2016 (Microsoft Corporation, USA). The principal component analysis (PCA) and hierarchical clustering analysis (HCA) were performed on the STATISTICA 12 (Dell Software, United States) and R package (R Foundation for Statistical Computing, Vienna, Austria), respectively.

\section{Results and Discussion}

3.1. Optimization of HPLC-DAD Conditions. An optimized strategy of the HPLC-DAD system was carried out to obtain 


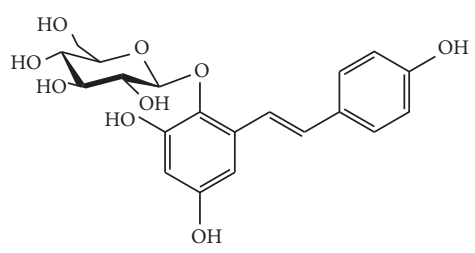

(a)

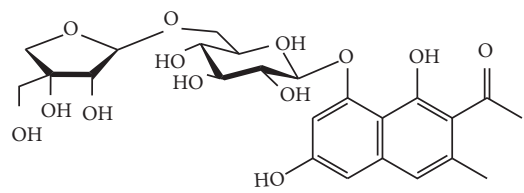

(e)

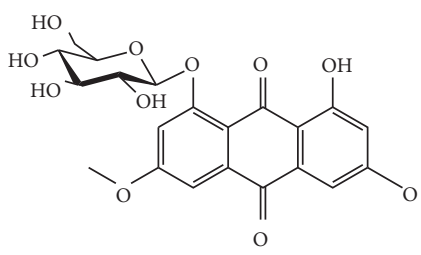

(b)

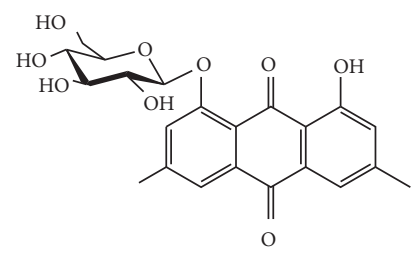

(c)

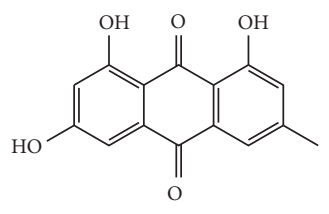

(d)

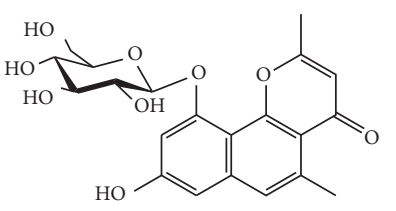

(f)

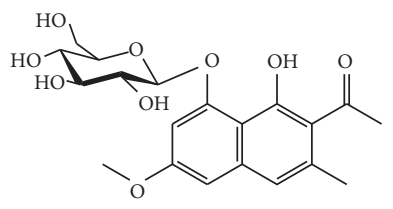

(g)

FIgURE 1: Chemical structures of seven standard compounds. (a) 2,3,4',5-tetrahydroxystilbene 2-O- $\beta$-D-glucopyranoside. (b) Physcionin. (c) Emodin 8-glucoside. (d) Emodin. (e) 6-hydroxymusizin 8-O- $\alpha$-D-apiofuranapiofuranosyl-(1-->6)- $\beta$-D-glucopyranoside. (f) Pleuropyrone A. (g) Torachrysone 8-O- $\beta$-D-glucopyranoside.

the separated peaks of the compounds on the chromatogram (Figure 2). All analytes were analyzed on the Agilent Series 1260 system using a ZORBAX Eclipse XDB C18 column (150 $\mathrm{mm} \times 4,6 \mathrm{~mm}, 5 \mu \mathrm{m})$ equipped with a ZORBAX Eclipse $\mathrm{XDB}$ C18 guard column $(12.5 \mathrm{~mm} \times 4.6 \mathrm{~mm}, 5 \mu \mathrm{m})$ kept at $30^{\circ} \mathrm{C}$. A mixture of $0.1 \% \mathrm{v} / \mathrm{v}$ of acetic acid in water and methanol with a linear gradient exhibited a better separation and peak shapes of all analytes. The flow rate was set at $0.5 \mathrm{ml} / \mathrm{min}$ with a linear gradient from 30 to $100 \%$ methanol over $40 \mathrm{~min}$, followed by $5 \mathrm{~min}$ washing with $100 \%$ methanol and $5 \mathrm{~min}$ of the initial condition. The injection volume for each sample was $5 \mu$ l. The UV detector was simultaneously set at $320 \mathrm{~nm}$ (for determination of compounds (1), (5), and (7)) and $280 \mathrm{~nm}$ (to identify compounds (2), (3), (4), and (6)). The retention time (Rt) of each compound is shown in Table 1.

3.2. Validation of the Method. The calibration curve of each compound was established from at least six appropriate concentrations in triplicate by plotting the peak areas versus the concentrations. As can be seen in Table 2, all calibration curves showed optimized linearities with the average determination coefficient $\left(R^{2}\right)$ higher than 0.999. LOD values were determined between 0.4 to $6.6 \mu \mathrm{g} / \mathrm{ml}$ illustrating a high sensitivity of the analytical method that was sufficient to determine the contents of $F$. multiflora samples.

The results of precision and accuracy were shown in Table 1 . The intraday and interday precision $(\mathrm{CV} \%)$ values ranged from $0.05 \%$ to $3.5 \%$, whereas the accuracy values calculated from recovery distributed between $98 \%$ and $103 \%$. These results showed acceptable precision and accuracy which were complying with the FDA guidance on bioanalytical methods [25]. Therefore, the established method is suitable for the simultaneous quantitation of selected compounds in F. multiflora.

3.3. Metabolomics and Quantification of F. Multiflora Samples. Metabolomics was utilized to identify the similarity and dissimilarity among a large number of samples. In this study, peak areas of seven compounds in 18 samples were collected from the chromatograms at the selected wavelengths and transferred to the tablet form to conduct hierarchical cluster analysis (HCA) and principal component analysis (PCA) for the discrimination of natural and commercial sliced F. multiflora. HCA was performed based on differences in contents of seven compounds which were represented by peak area values. The Euclidean distances among 18 samples were calculated on R package software that was illustrated on the HCA dendrogram (Figure 3). As can be seen, the group of six natural samples showed a dramatic difference to the commercial sliced samples on the HCA dendrogram. Meanwhile, the commercial medicinal sliced samples clearly clustered among the samples CMS_06, CMS_08 to CMS_10, CMS_11 to CMS_12, and the rest of the CMS samples.

On the other hand, the PCA score plot (Figure 4(a)) was also sharply divided into two regions represented natural samples and other commercial samples, whereas the CMS samples were also separated into four clusters on the PCA plot, which were similar on the HCA dendrogram. As can be seen from the scree plot (Figure 5), the first two principal components (PCs) accounted for more than $90 \%$ of the total variation of samples. Thus, we suppose that these PCs carried major information of variables. Considerably, CMS_06 was determined as a specific sample on the PCA plot. These results indicated a clear difference in the contents of standard compounds between natural and commercial products of $F$. multiflora. Furthermore, CMS samples could be categorized into four CMS groups: group 1: CMS_06; group 2: CMS_08 to CMS_10; group 3: CMS_11 to CMS_12; group 4: rest of CMS samples, based on the results of PCA and HCA. Marker compounds for the clustering could be identified on the basis of the PCA loading plot (Figure 4(b)). As can be seen, emodin (4), THSG (1), and 6-hydroxymusizin $\quad 8-O-\alpha$-D-apiofuranosyl-( $(\longrightarrow 6)-\beta$-D-glucopyranoside (5) could be clearly detected as marker compounds to distinguish F. multiflora samples.

For a further explanation of the discrimination among the samples, contents of seven selected standards were 


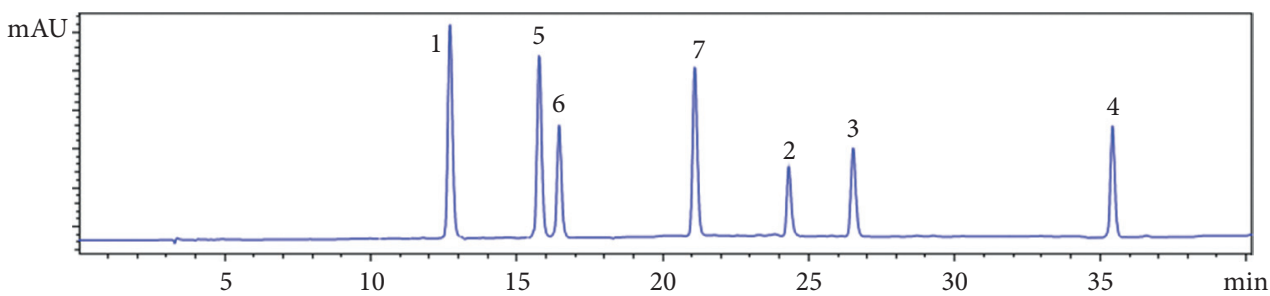

(a)

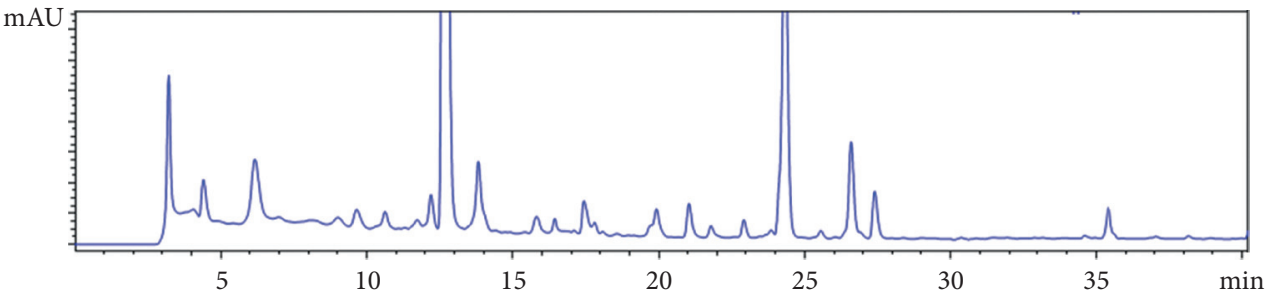

(b)

FIGURE 2: The representative chromatograms at UV $280 \mathrm{~nm}$ of (a) a standards mixture and (b) the sample Nat_01. The numbers in brackets indicate seven standard compounds as mentioned above.

TABLE 1: Intra- and Interday precision and accuracy of seven standard compounds in F. multiflora.

\begin{tabular}{|c|c|c|c|c|c|c|}
\hline \multirow[b]{2}{*}{ Analyte } & \multirow[b]{2}{*}{ Rt (min) } & \multirow[b]{2}{*}{$\mathrm{C}(\mu \mathrm{g} / \mathrm{ml})$} & \multicolumn{2}{|r|}{ Intraday $(n=6)$} & \multicolumn{2}{|r|}{ Interday $(n=6)$} \\
\hline & & & $\begin{array}{l}\text { Precision } \\
(\mathrm{CV} \%)\end{array}$ & Accuracy (\% from recovery) mean $\pm S D$ & $\begin{array}{l}\text { Precision } \\
(\mathrm{CV} \%)\end{array}$ & $\begin{array}{c}\text { Accuracy (\% from recovery) } \\
\text { mean } \pm \text { SD }\end{array}$ \\
\hline \multirow{3}{*}{ (1) } & \multirow{3}{*}{12.7} & 25 & 0.7736 & $100.19 \pm 4.98$ & 1.7772 & $98.68 \pm 8.35$ \\
\hline & & 125 & 0.1587 & $98.68 \pm 2.36$ & 0.6515 & $100.64 \pm 2.17$ \\
\hline & & 500 & 0.9496 & $99.49 \pm 0.82$ & 0.8775 & $99.61 \pm 1.95$ \\
\hline \multirow{3}{*}{ (2) } & \multirow{3}{*}{24.3} & 25 & 1.7564 & $99.94 \pm 4.82$ & 1.6769 & $100.86 \pm 5.14$ \\
\hline & & 125 & 0.1958 & $99.93 \pm 2.04$ & 0.7853 & $99.82 \pm 2.04$ \\
\hline & & 500 & 0.0788 & $99.97 \pm 0.32$ & 0.1215 & $100.42 \pm 0.57$ \\
\hline \multirow{3}{*}{ (3) } & \multirow{3}{*}{26.5} & 25 & 1.0490 & $99.38 \pm 4.03$ & 1.4874 & $99.28 \pm 3.3$ \\
\hline & & 125 & 0.5440 & $98.4 \pm 0.99$ & 0.5175 & $98.9 \pm 0.94$ \\
\hline & & 500 & 0.2066 & $99.48 \pm 0.49$ & 0.2465 & $98.97 \pm 0.54$ \\
\hline \multirow{3}{*}{ (4) } & \multirow{3}{*}{35.3} & 25 & 0.3930 & $98.37 \pm 2.6$ & 0.5160 & $98.42 \pm 5.16$ \\
\hline & & 125 & 0.0768 & $98.58 \pm 0.91$ & 0.2293 & $99.27 \pm 1.22$ \\
\hline & & 500 & 0.0671 & $100.05 \pm 0.42$ & 1.4881 & $100.52 \pm 0.64$ \\
\hline \multirow{3}{*}{ (5) } & \multirow{3}{*}{15.7} & 25 & 3.4528 & $99.64 \pm 3.12$ & 3.4721 & $102.99 \pm 6.06$ \\
\hline & & 125 & 1.2993 & $100.71 \pm 1.05$ & 1.2698 & $98.1 \pm 2.73$ \\
\hline & & 500 & 0.9496 & $100.13 \pm 0.29$ & 0.7438 & $103.09 \pm 0.54$ \\
\hline \multirow{3}{*}{ (6) } & \multirow{3}{*}{16.4} & 25 & 1.8823 & $98.23 \pm 4.37$ & 1.7781 & $99.05 \pm 4.42$ \\
\hline & & 125 & 1.2309 & $100.56 \pm 1.27$ & 1.2267 & $101.02 \pm 1.40$ \\
\hline & & 500 & 0.3624 & $99.8 \pm 0.41$ & 0.3514 & $99.97 \pm 0.43$ \\
\hline \multirow{3}{*}{ (7) } & \multirow{3}{*}{21.0} & 25 & 2.2353 & $100.45 \pm 4.99$ & 2.0892 & $98.7 \pm 8.22$ \\
\hline & & 125 & 1.3567 & $99.54 \pm 1.78$ & 1.1548 & $99.54 \pm 1.78$ \\
\hline & & 500 & 0.9318 & $99.77 \pm 0.25$ & 0.7520 & $98.75 \pm 0.80$ \\
\hline
\end{tabular}

TABLe 2: Regression data for all standards with LOD and LOQ.

\begin{tabular}{lccccc}
\hline Analyte & Slope $^{*}$ & Intercept & $R^{2}$ & LOD $(\mu \mathrm{g} / \mathrm{ml})$ & LOQ $(\mu \mathrm{g} / \mathrm{ml})$ \\
\hline $\mathbf{( 1 )}$ & 6.1379 & 3.9659 & 0.9999 & 0.5106 & 1.5474 \\
$(\mathbf{2})$ & 4.0861 & 21.5671 & 0.9998 & 0.4997 & 1.5143 \\
$(\mathbf{3})$ & 2.0617 & 9.3186 & 0.9996 & 0.7349 & 2.2269 \\
$(\mathbf{4})$ & 6.6230 & -35.4954 & 0.9998 & 6.6000 & 19.8000 \\
$(\mathbf{5})$ & 1.0218 & 0.7870 & 0.9999 & 1.3600 & 4.1212 \\
$(\mathbf{6})$ & 2.3317 & 6.8226 & 0.9999 & 1.4333 & 4.3435 \\
$(7)$ & 0.9279 & 2.4082 & 0.9998 & 1.5736 & 4.7684 \\
\hline
\end{tabular}

${ }^{*}$ Experiments were performed in triplicate. quantified using the method above. The results were calculated and summarized in Table 3.

The contents of each compound varied considerably in different F. multiflora samples. THSG (1) contents in natural samples ranged from 26,211 to $55,010 \mathrm{mg} / \mathrm{kg}$, which were 4 to 108 -fold higher than in CMS samples. THSG contents also varied widely among CMS groups as categorized above. CMS group 2 contained the highest contents of THSG among four CMS groups at more than $9,000 \mathrm{mg} / \mathrm{kg}$, followed by CMS group 3 at about $5,000 \mathrm{mg} / \mathrm{kg}$, whereas THSG 


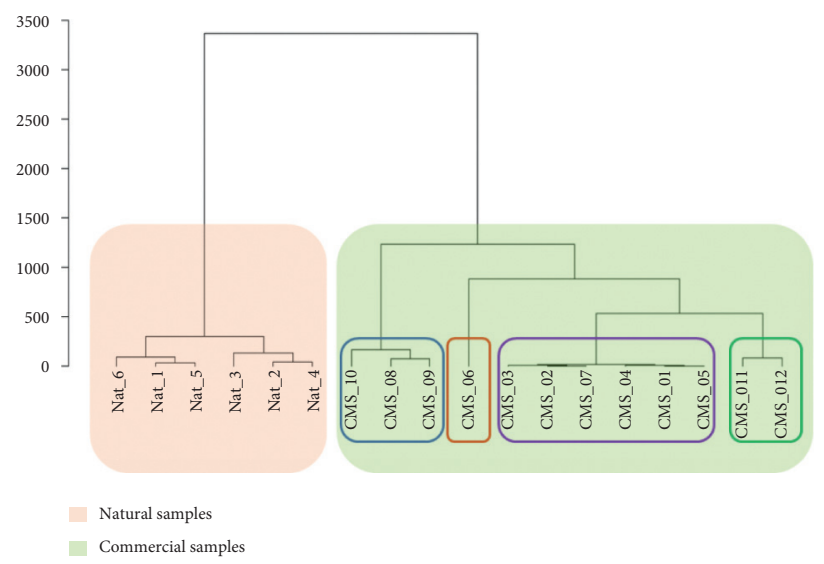

Figure 3: HCA dendrogram of natural and commercial F. multiflora samples with detected metabolites.

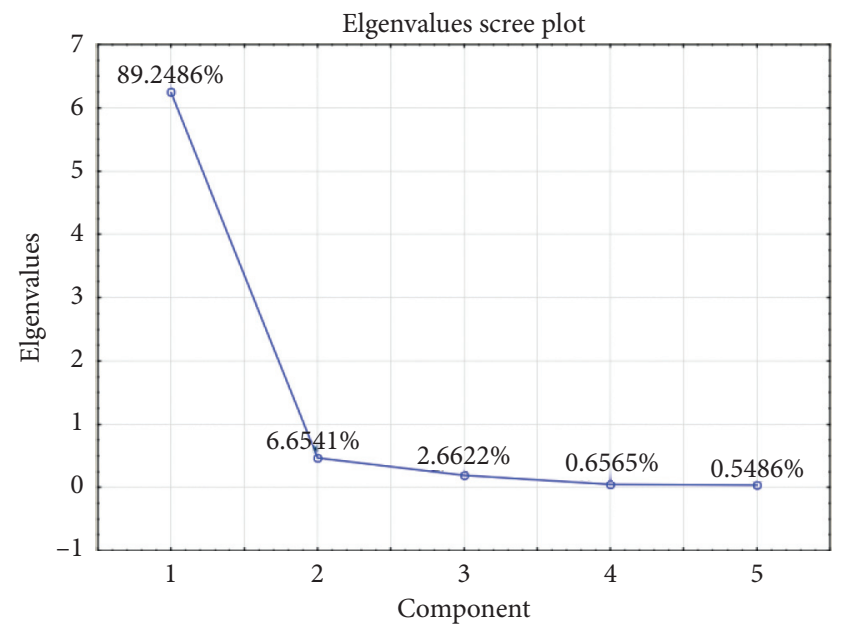

Figure 4: PCA scree plot.

contents of the sample CMS_01 to CMS_07 (CMS groups 1 and 4) varied at low levels, from 507 to $3,285 \mathrm{mg} / \mathrm{kg}$. Previous studies also reported that THSG contents may range from 8,000 to $60,000 \mathrm{mg} / \mathrm{kg}$ of natural $F$. multiflora, and at sharply lower levels in commercial samples [16, 18, 21]. Contents of the compound, 6-hydroxymusizin $8-O-\alpha$-D-

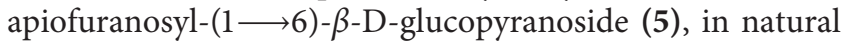
samples were dramatically higher than other CMS samples, while CMS group 2 showed the highest content of the compound among four CMS groups. Considerably, very low or no contents of physcionin (2), emodin 8-glucoside (3), pleuropyrone A (6), and torachrysone $8-O-\beta$-D-glucopyranoside (7) were determined in samples of CMS group 1 and 4 , while natural samples showed the highest contents of these metabolites, which were at least 3-fold higher than the values of CMS groups 2 and 3. Interestingly, emodin (4) was found at the highest level in the sample CMS_06 (CMS group 1), followed by natural samples, CMS group 2, and CMS group 3, respectively, while detected at low contents in CMS group 4. This compound might be useful as a marker that led to the clear separation of the sample CMS_06 to the other analytes on the PCA score plot. A study on processing F. multiflora illustrated that the emodin content may increase $20-30 \%$ after steaming with or without black beans for a long time [15]. Thus, CMS_06 might be processed in a related condition which sharply increased the synthesis of emodin.

Overall, except emodin, six other standards showed higher contents in natural samples that might lead to discrimination to CMS samples. The clusters of samples in CMS groups could base on the different contents of THSG (1), physcionin (2), emodin 8-glucoside (3), 6-hydroxymusizin 8 -O- $\alpha$-D-apiofuranosyl-(1 $\longrightarrow 6)-\beta$-D-glucopyranoside $\quad(5)$, pleuropyrone A (6), and torachrysone $8-O-\beta$-D-glucopyranoside (7), where CMS group 2 contained higher contents of standards than CMS groups 3 and 4, respectively. CMS group 1, included only the sample CMS_06, was classified by a significantly high content of emodin (4). Considerably, the variation in contents of 6-hydroxymusizin $8-O-\alpha$-D-apio-

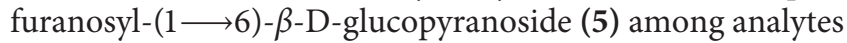
showed a similar trend as THSG (1), thus, could be used as a new marker for classification of F. multiflora. In general, commercial F. multiflora products were processed by steaming with black bean or stored under unappropriated conditions, which notably decreased the contents of metabolites [9]. The previous study reported that and $50 \%$ of stilbenes contents might decompose after high-temperature processing, which reduced sharply the toxicity of F. multiflora [15]. However, THSG and emodin were proved as the major anticancer, antioxidant, and antivirus agents of F. multiflora [25-36]. Thus, the decrease in these compounds may lead to a serious decline in the quality of medicine. In addition, the obtained results showed a variation of THSG contents in CMS samples, in which the contents of THSG in natural samples may be up to 108 -fold higher than CMS samples. A previous study on F. multiflora also illustrated a high difference in the contents of major metabolites between natural and commercial processed products. Accordingly, THSG or emodin contents of natural roots might be up to 130 -fold higher than in commercial products [22]. The variation of compositions of CMS samples in this study indicated that the quality of commercial F. multiflora may vary significantly among manufactures due to different processing or preserving methods. Therefore, further studies should be carried out to explain clearly the reason and consequences of these issues. 


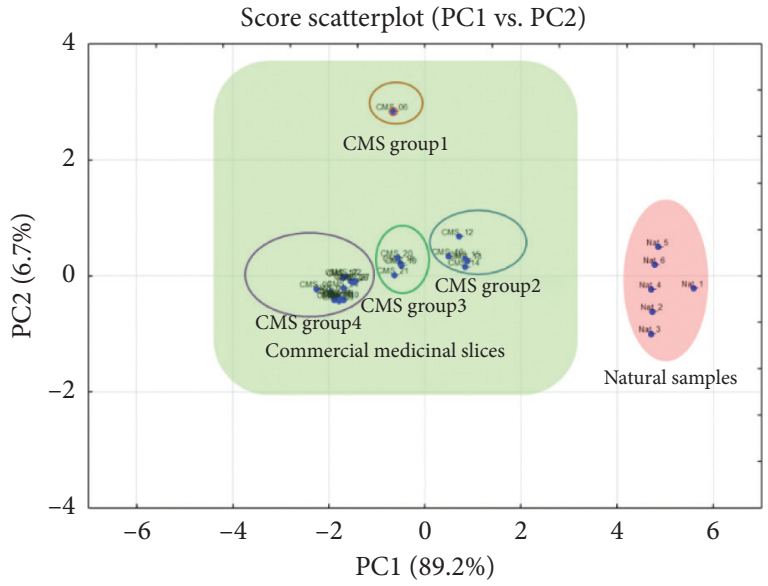

(a)

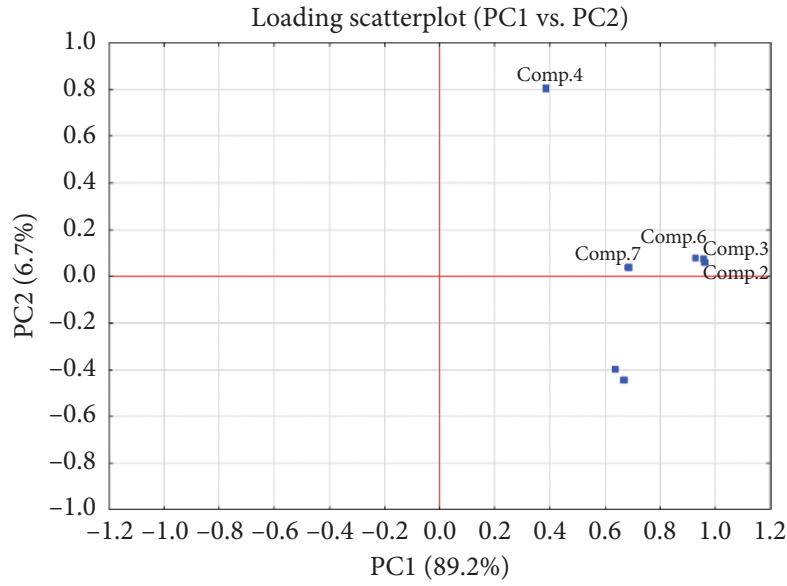

(b)

FIgURE 5: PCA 2D score plot (a) and loading plot (b) of natural and commercial $F$ multiflora samples with detected metabolites.

TABLE 3: The contents of seven compounds in eighteen F. multiflora samples.

\begin{tabular}{|c|c|c|c|c|c|c|c|c|}
\hline \multirow{2}{*}{ Group* } & \multirow{2}{*}{ Sample } & \multicolumn{7}{|c|}{ Content (mg/kg dry weight)** } \\
\hline & & (1) & $(2)$ & (3) & $(4)$ & $(5)$ & $(6)$ & $(7)$ \\
\hline \multirow{6}{*}{ Natural samples } & Nat_1 & $55010 \pm 2525$ & $3183 \pm 135.5$ & $1404 \pm 72.59$ & $438.13 \pm 14.09$ & $173.4 \pm 9.13$ & $117.0 \pm 6.23$ & $398.0 \pm 17.03$ \\
\hline & Nat_2 & $46946 \pm 3188$ & $3175 \pm 234.3$ & $1368 \pm 48.97$ & $333.5 \pm 14.54$ & $148.7 \pm 4.99$ & $100.6 \pm 3.34$ & $383.5 \pm 9.04$ \\
\hline & Nat_3 & $50491 \pm 2826$ & $3170 \pm 155.2$ & $1399 \pm 86.74$ & $268.3 \pm 14.37$ & $170.5 \pm 10.84$ & $115.5 \pm 7.32$ & $342.1 \pm 11.49$ \\
\hline & Nat_4 & $33660 \pm 2393$ & $3190 \pm 260.3$ & $1405 \pm 53.36$ & $354.2 \pm 23.93$ & $149.6 \pm 7.12$ & $117.3 \pm 5.25$ & $411.3 \pm 23.68$ \\
\hline & Nat_5 & $29176 \pm 1923$ & $3199 \pm 216.2$ & $1386 \pm 86.71$ & $457.9 \pm 14.92$ & $155.7 \pm 5.07$ & $131.4 \pm 4.63$ & $361.7 \pm 22.64$ \\
\hline & Nat_6 & $26211 \pm 678.9$ & $3223 \pm 192.0$ & $1375 \pm 35.47$ & $404.8 \pm 26.64$ & $157.8 \pm 8.81$ & $114.6 \pm 6.50$ & $459.6 \pm 21.05$ \\
\hline CMS group 1 & CMS_06 & $1698 \pm 111.9$ & + & + & $547.0 \pm 25.65$ & $35.73 \pm 1.30$ & + & ND \\
\hline \multirow{6}{*}{ CMS group 4} & CMS_01 & $1170 \pm 66.92$ & ND & + & + & $20.41 \pm 0.97$ & + & + \\
\hline & CMS_02 & $2160 \pm 207.1$ & + & ND & + & $25.64 \pm 0.58$ & + & + \\
\hline & CMS_03 & $507.0 \pm 23.27$ & + & + & + & ND & ND & ND \\
\hline & CMS_04 & $2285 \pm 145.3$ & + & + & + & $43.37 \pm 1.55$ & ND & ND \\
\hline & CMS_05 & $3285 \pm 282.2$ & + & + & + & $38.81 \pm 1.78$ & + & ND \\
\hline & CMS_07 & $2418 \pm 110.7$ & + & + & + & $39.40 \pm 1.45$ & ND & ND \\
\hline \multirow{3}{*}{ CMS group 2} & CMS_08 & $9094 \pm 435.1$ & $985.0 \pm 45.11$ & $326.6 \pm 14.96$ & $93.89 \pm 2.97$ & $62.67 \pm 2.61$ & $63.57 \pm 3.00$ & $219.2 \pm 11.33$ \\
\hline & CMS_09 & $11854 \pm 965.8$ & $907.2 \pm 32.28$ & $351.2 \pm 11.52$ & $104.3 \pm 3.48$ & $75.11 \pm 1.76$ & $71.00 \pm 2.01$ & $202.7 \pm 6.77$ \\
\hline & CMS_10 & $10470 \pm 692.1$ & $818.6 \pm 53.75$ & $340.7 \pm 24.57$ & $119.0 \pm 4.26$ & $90.48 \pm 3.93$ & $78.21 \pm 3.86$ & $198.3 \pm 4.65$ \\
\hline \multirow{2}{*}{ CMS group 3} & CMS_11 & $5335 \pm 298.2$ & $427.4 \pm 22.98$ & $234.3 \pm 3.68$ & $112.6 \pm 4.89$ & $34.81 \pm 1.51$ & $42.87 \pm 1.73$ & $139.6 \pm 7.46$ \\
\hline & CMS_12 & $4770 \pm 328.6$ & $508.9 \pm 40.50$ & $203.5 \pm 10.29$ & $99.67 \pm 1.28$ & $29.81 \pm 0.96$ & $52.27 \pm 2.05$ & $153.3 \pm 6.46$ \\
\hline
\end{tabular}

${ }^{*}$ Categorized by HCA and PCA. **Experiments were performed in triplicate. +: Identifiable under the limit of quantitation.; ND : not detected.

\section{Conclusions}

For the first time, an HPLC-DAD based metabolomics approach was performed to discriminate the natural and commercial medicinal slices of F. multiflora roots in Vietnam. Moreover, seven compositions were quantified to evaluate the quality of natural and commercial samples. With this approach, the natural F. multiflora samples were significantly distinguished from the commercial ones. Large different contents of seven selected metabolites among two categories of samples indicated dramatic changes of F. multiflora chemical compositions on processing and manufacturing. On the other hand, the discrimination based on the difference of standard metabolites of commercial samples performed a considerable variation of F. multiflora quality among manufactures. Because F. multiflora is a common traditional medicine, its quality needs to be strictly controlled. Besides emodin and THSG, interestingly, the new compound, 6-hydroxymusizin $8-O-\alpha$-D-apiofuranosyl$(1 \longrightarrow 6)-\beta$-D-glucopyranoside was identified as a marker for the discrimination. Overall, this study presented an effective method for precisely distinguishing natural and commercial F. multiflora products, also possibly applying for quality evaluation of other diverse medicinal herbs.

\section{Data Availability}

All data supporting the findings of this study are included within the article. 


\section{Conflicts of Interest}

The authors declare that there are no conflicts of interest regarding the publication of this paper.

\section{Acknowledgments}

This work was supported in part by the Vietnam Academy of Science and Technology (Grant code VAST.UDCN.05/ 14-16).

\section{References}

[1] Vietnam Ministry of Medicines, Vietnamese Pharmacopoeiap. 1180, 5th edition, Ho Chi Minh, Vietnam, 2017.

[2] E.-Y. Lin, A. Chagnaadorj, S.-J. Huang, C.-C. Wang, Y.-H. Chiang, and C.-W. Cheng, "Hepatoprotective activity of the ethanolic extract of polygonum multiflorum thunb. Against oxidative stress-induced liver injury," Evidence-Based Complementary and Alternative Medicine, vol. 2018, Article ID 4130307, 9 pages, 2018.

[3] X. Wang, L. Zhao, T. Han, S. Chen, and J. Wang, "Protective effects of 2,3,5,4'-tetrahydroxystilbene-2-O-beta-D-glucoside, an active component of Polygonum multiflorum Thunb, on experimental colitis in mice," European Journal of Pharmacology, vol. 578, no. 2-3, pp. 339-348, 2008.

[4] H. Q. Lin, M. T. Ho, L. S. Lau, K. K. Wong, P. C. Shaw, and D. C. C. Wan, "Anti-acetylcholinesterase activities of traditional Chinese medicine for treating Alzheimer's disease," Chemico-Biological Interactions, vol. 175, no. 1-3, pp. 352-354, 2008.

[5] L. Zhang, L. Huang, L. Chen, D. Hao, and J. Chen, "Neuroprotection by tetrahydroxystilbene glucoside in the MPTP mouse model of Parkinson's disease," Toxicology Letters, vol. 222, no. 2, pp. 155-163, 2013.

[6] F. W.-K. Cheung, A. W.-N. Leung, W. K. Liu, and C.-T. Che, "Tyrosinase inhibitory activity of a glucosylated hydroxystilbene in mouse melan-a melanocytes," Journal of Natural Products, vol. 77, no. 6, pp. 1270-1274, 2014.

[7] L. Lv, X. Gu, J. Tang, and C. Ho, "Antioxidant activity of stilbene glycoside from Polygonum multiflorum thunb in vivo," Food Chemistry, vol. 104, no. 4, pp. 1678-1681, 2014.

[8] P. P. Toth, "Drug treatment of hyperlipidaemia," Drugs, vol. 70, no. 11, pp. 1363-1379, 2010.

[9] L. Lin, B. Ni, H. Lin et al., "Traditional usages, botany, phytochemistry, pharmacology, and toxicology of Polygonum multiflorum thunb: a review," Journal of Ethnopharmacology, vol. 159, pp. 158-183, 2015.

[10] Y. Liu, Q. Wang, J. Yang et al., "Polygonum multiflorum thunb: a review on chemical analysis, processing mechanism, quality evaluation, and hepatotoxicity," Frontiers in Pharmacology, vol. 9364 pages, 2018.

[11] L.-L. Chen, X.-J. Huang, M.-M. Li et al., "Polygonflavanol a, a novel flavonostilbene glycoside from the roots of Polygonum multiflorum," Phytochemistry Letters, vol. 5, no. 4, pp. 756-760, 2012.

[12] M.-H. Kim, J. H. Park, H. Won, and C.-W. Park, "Flavonoid chemistry and chromosome numbers of fallopia section Pleuropterus (Polygonaceae)," Canadian Journal of Botany, vol. 78, no. 9, pp. 1136-1143, 2000.

[13] Y. Chen, M. Wang, R. T. Rosen, and C.-T. Ho, "2,2-Diphenyl1-picrylhydrazyl radical-scavenging active components from
Polygonum multiflorum thunb," Journal of Agricultural and Food Chemistry, vol. 47, no. 6, pp. 2226-2228, 1999.

[14] H. N. Kim, Y. R. Kim, J. Y. Jang et al., "Neuroprotective effects of Polygonum multiflorum extract against glutamate-induced oxidative toxicity in HT22 hippocampal cells," Journal of Ethnopharmacology, vol. 150, no. 1, pp. 108-115, 2013.

[15] X. Wu, X. Chen, Q. Huang, D. Fang, G. Li, and G. Zhang, "Toxicity of raw and processed roots of Polygonum multiflorum," Fitoterapia, vol. 83, no. 3, pp. 469-475, 2012.

[16] H. F. Chen, Y. H. Chen, C. H. Liu et al., "Integrated chemometric fingerprints of antioxidant activities and HPLC-DAD-CL for assessing the quality of the processed roots of Polygonum multiflorum Thunb. (Heshouwu)," Chinese Medicine, vol. 11, p. 18, 2016.

[17] N. Li, Z. Chen, X. Mao, J. Yu, and R. Zhao, "Effects of lipid regulation using raw and processed Radix Polygoni Multiflori in rats fed a high-fat diet," Evidence-Based Complementary and Alternative Medicine, vol. 2012, Article ID 329171, 10 pages, 2012.

[18] T.-H. Wang, J. Zhang, X.-H. Qiu, J.-Q. Bai, Y.-H. Gao, and $\mathrm{W}$. $\mathrm{Xu}$, "Application of ultra-high-performance liquid chromatography coupled with LTQ-orbitrap mass spectrometry for the qualitative and quantitative analysis of $\mathrm{Po}$ lygonum multiflorum thumb. and its processed products," Molecules, vol. 21, no. 1, p. 40, 2015.

[19] L. Lin, H. Li, H. Lin et al., "A new perspective on liver injury by traditional Chinese herbs such as Polygonum multiflorum: the geographical area of harvest as an important contributory factor," Frontiers in Pharmacology, vol. 8, p. 349, 2017.

[20] X. Qiu, J. Zhang, Z. Huang, D. Zhu, and W. Xu, "Profiling of phenolic constituents in Polygonum multiflorum thunb. by combination of ultra-high-pressure liquid chromatography with linear ion trap-orbitrap mass spectrometry," Journal of Chromatography A, vol. 1292, pp. 121-131, 2013.

[21] T. Kang, Z. Zhao, and L. Liang, "Application of microscopy technique and high performance liquid chromatography for quality assessment of Polygonum multiflorum thunb. (Heshouwu)," Pharmacognosy Magazine, vol. 10, no. 40, p. 415, 2014.

[22] Y. Zhao, C. P. Kao, Y. S. Chang, and Y. L. Ho, "Quality assessment on Polygoni Multiflori Caulis using HPLC/UV/ MS combined with principle component analysis," Chemistry Central Journal, vol. 7, no. 1, p. 106, 2013.

[23] V. C. Vo, Dictionary of Vietnamese Medicinal Plants, Vol. I, Medicine Publisher, Hanoi, Vietnam, 2012.

[24] T. T. Nguyen, T. B. Pham, N. P. Thao et al., "Phenolic constituents from Fallopia multiflora (thunberg) Haraldson," Journal of Chemistry, vol. 2018, Article ID 4851439, 5 pages, 2018.

[25] Food and Drug Administration (FDA), Center for Drug Evaluation and Research, Bioanalytical Methods Validation: Guidance for Industry, US Department of Health and Human Services, Washington, D.C USA, 2018.

[26] T.-T. A. Nguyen, M. T. Ha, S.-E. Park, J. S. Choi, B. S. Min, and J. A. Kim, "Stilbenes with potent protein tyrosine phosphatase-1B inhibitory activity from the roots of Polygonum multiflorum," Journal of Natural Products, vol. 83, no. 2, pp. 323-332, 2020.

[27] P.-W. Tsai, Y.-H. Lee, L.-G. Chen, C.-J. Lee, and C.-C. Wang, "In vitro and in vivo anti-osteoarthritis effects of $2,3,5,4$ " tetrahydroxystilbene-2-O- $\beta$-d-Glucoside from Polygonum multiflorum," Molecules, vol. 23, no. 3, p. 571, 2018.

[28] X. Xu, Q. Zhu, C. Zhao et al., "The effect of 2,3,4',5-tetrahydroxystilbene-2-O- $\beta$-D-Glucoside on pressure overload- 
induced cardiac remodeling in rats and its possible mechanism," Planta Medica, vol. 80, no. 2/3, pp. 130-138, 2014.

[29] T. Ho, S. Wu, J. Chen, C. Li, and C. Hsiang, "Emodin blocks the SARS coronavirus spike protein and angiotensin-converting enzyme 2 interaction," Antiviral Research, vol. 74, no. 2, pp. 92-101, 2007.

[30] K.-M. Lim, J.-H. Kwon, K. Kim et al., "Emodin inhibits tonic tension through suppressing $\mathrm{PKC} \delta$-mediated inhibition of myosin phosphatase in rat isolated thoracic aorta," British Journal of Pharmacology, vol. 171, no. 18, pp. 4300-4310, 2014.

[31] C.-M. Hsu, Y.-A. Hsu, Y. Tsai et al., "Emodin inhibits the growth of hepatoma cells: finding the common anti-cancer pathway using Huh7, Hep3B, and HepG2 cells," Biochemical and Biophysical Research Communications, vol. 392, no. 4, pp. 473-478, 2010.

[32] D. Zhou, B. Gao, Y. Kong, and W. Wang, "Integrative analysis of ceRNA network reveals functional lncRNAs in intrahepatic cholangiocarcinoma," BioMed Research International, vol. 2019, Article ID 2601271, 14 pages, 2019.

[33] S. Masaldan and V. V. Iyer, "Exploration of effects of emodin in selected cancer cell lines: enhanced growth inhibition by ascorbic acid and regulation of LRP1 and AR under hypoxialike conditions," Journal of Applied Toxicology, vol. 34, no. 1, pp. 95-104, 2012.

[34] Y. Zheng, J. Li, J. Wu et al., "Tetrahydroxystilbene glucoside isolated from Polygonum multiflorum thunb. demonstrates osteoblast differentiation promoting activity," Experimental and Therapeutic Medicine, vol. 14, no. 4, pp. 2845-2852, 2017.

[35] G. P. Lv, L. Z. Meng, D. Q. Han, H. Y. Li, J. Zhao, and S. P. Li, "Effect of sample preparation on components and liver toxicity of Polygonum multiflorum," Journal of Pharmaceutical and Biomedical Analysis, vol. 109, pp. 105-111, 2015.

[36] G. A. Bounda and Y. U. Feng, "Review of clinical studies of Polygonum multiflorum thunb. and its isolated bioactive compounds," Pharmacognosy Research, vol. 7, no. 3, pp. 225-236, 2015. 